\title{
Conocimientos sobre prácticas de autocuidado y su asociación con las características sociodemográficas de pacientes con diagnóstico de tuberculosis pulmonar, CLAS San Martín de Porres - Los Olivos - 2012
}

\author{
Aliaga-Rojas Gloria ${ }^{1}$, Alvarado-Herrera María1, Alva-Vera Ángela1, Velásquez-Carranza Doris ${ }^{2}$
}

\section{RESUMEN}

Objetivo: determinar el nivel de conocimientos sobre prácticas de autocuidado y su relación con las variables sociodemográficas en pacientes con tuberculosis pulmonar registrados en la estrategia nacional de prevención y control de tuberculosis pulmonar. Material y métodos: el tipo de estudio es descriptivo de corte transversal. La población estuvo conformada por 30 pacientes con diagnóstico de tuberculosis pulmonar pertenecientes al esquema de tratamiento I, que se encuentran registrados en la Estrategia Nacional de Prevención y Control de Tuberculosis Pulmonar del Centro de Salud CLAS «San Martín de Porres». Se utilizó como técnica la encuesta y como instrumento el cuestionario estructurado, elaborado por las investigadoras. El instrumento fue validado por juicio de expertos y se realizó la prueba piloto. Para el procesamiento de datos se utilizó el programa estadístico SPSS versión 15.0, para la recolección de datos se solicitó el permiso a la institución, se identificó a la población de estudio y se solicitó el consentimiento informado. Resultados: los resultados mostraron que, del total de pacientes evaluados predominó el nivel de conocimientos medio con 43,3\%, mientras que 26,7\% de los pacientes presentó un nivel de conocimientos bajo. El $43 \%$ de los pacientes de sexo femenino presentó un alto nivel de conocimientos. El $50 \%$ de pacientes entre 25 a 44 años presentó un nivel de concimiento medio. Las pacientes de nivel de educación superior presentan un nivel de conocimiento alto; con relación al grado de instrucción el que predominó fue superior universitario con 50\%; el 45\% de pacientes que trabajan presentó un nivel de conocimientos alto. Se respetaron los principios bioéticos. Conclusiones: la mayoría de los pacientes con tuberculosis presentan un nivel de conocimiento medio bajo sobre autocuidado. Existe asociación significativa entre el nivel de conocimiento al nivel de instrucción y la ocupación de los pacientes. No se encontró asociación significativa entre conocimientos de prácticas de autocuidado con la edad y sexo. Existe asociación significativa entre el nivel de conocimientos de prácticas de autocuidado con el grado de instrucción y ocupación.

Palabras clave: conocimiento, autocuidado, tuberculosis pulmonar. Perú. (Fuente DeCs BIREME).

\section{Practical knowledge of self and its association with demographic characteristics in patients with pulmonary tuberculosis, San Martin de Porres CLAS - Los Olivos - 2012}

\begin{abstract}
Objetive: Determine the level of knowledge about self-care practices and their relationship with sociodemographic variables in patients with pulmonary tuberculosis registered in the national strategy for prevention and control of pulmonary tuberculosis. Material and Methods: Type of study is a descriptive cross sectional. The population consisted of 30 patients diagnosed with pulmonary tuberculosis belonging to the treatment schedule I, which are recorded in the National Strategy for Prevention and Control of Tuberculosis Lung Health Center CLAS «San Martin de Porres». The survey was used as a technique and a tool structured questionnaire developed by the researchers. The instrument was validated by expert opinion and pilot testing was performed. For data processing SPSS version 15.0 was used for data
\end{abstract}

1. Egresada de Enfermería de la Universidad Peruana Cayetano Heredia. Lima - Perú.

2. Magister en Enfermería. Profesora Asociada de la Facultad de Enfermería. Universidad Peruana Cayetano Heredia. Lima - Perú. 
collection to the institution permission was sought, identified the study population and informed consent was requested. Results: The results showed that, of the patients evaluated predominant medium level knowledge with $43.3 \%$, while $26.7 \%$ of patients had a low level of knowledge. $43 \%$ of the female patients had a high level of knowledge. $50 \%$ of patients between 25 and 44 years presented a medium concimiento. Patients with higher level of education have a higher level of knowledge, relative to the level of education that prevailed was the top university with $50 \%, 45 \%$ of working patients presented a higher level of knowledge. Bioethical principles are respected.Conclusions. most patients with tuberculosis have lower average level of knowledge about self-care. There is significant association between the level of knowledge to the level of education and occupation of patients. No significant association between knowledge of self-care practices to age and sex was found. There is significant association between the level of knowledge of self-care practices with the level of education and occupation.

Key words: Knowledge, self-care, pulmonary tuberculosis. Peru. (Source DeCs BIREME).

\section{INTRODUCCIÓN}

La tuberculosis es una enfermedad infectocontagiosa, considerada un problema de salud pública en todo el mundo, lo que se evidencia en las altas tasas de morbilidad y mortalidad. Un tercio de la población mundial está actualmente infectada y cada segundo se produce en el mundo una nueva infección por el bacilo de la tuberculosis (1). En el año 2008, 9,4 millones de personas contrajeron la enfermedad, y 1,8 millones fallecieron. En promedio, cada año en el mundo ocurren entre 8 y 9 millones de casos y son causa de 2 a 3 millones de muertes anualmente (2).

La Organización Mundial de Salud indica que en el año 2009 en el Perú se han notificado 17989 casos nuevos mientras que a nivel mundial los casos ascienden a 2654410 habitantes. La tuberculosis sigue siendo un problema de salud pública de importancia por la magnitud de los daños que se evidencian en la morbilidad y mortalidad de la población (3).

Según la Organización Mundial de la Salud el Perú tiene, después de Haití, la tasa más alta de tuberculosis de Latinoamérica. En el último año se contabilizaron 33 mil casos de tuberculosis sensible, de los cuales el $59 \%$ se presenta en Lima (4).

La función primordial del enfermero (a) es el cuidado de los individuos en el proceso salud-enfermedad, es muy importante hacer énfasis en los cuidados brindados a la población, esto se logra a través de la puesta en marcha de la promoción de la salud, dentro de esta, la enfermera aporta elementos a los individuos para que se empoderen de su propio cuidado y así mejoren su calidad de vida a través del autocuidado. El autocuidado es necesario para la búsqueda del bienestar integral en la vida cotidiana y el desarrollo humano. Por lo tanto, el autocuidado para el manejo de la tuberculosis pulmonar es una práctica que involucra líneas de crecimiento en las que toda persona debe trabajar cotidianamente para tener un desarrollo armónico y equilibrado en el control de su enfermedad. Con el fin de lograr esto, es importante conocer los aspectos sociales, culturales y sociodemográficas de la población en la que se aplican para lograr pertinencia, cambio y empoderamiento de sus prácticas saludables y mejorar su estilo de vida (5).

Se ha reconocido que la tuberculosis pulmonar está ligada a la pobreza, tal y como lo evidencia su distribución a nivel mundial. Los países subdesarrollados son los más afectados, no solamente por el número de casos, sino por las pérdidas en las horas de trabajo y en la productividad (4). Esta situación es más crítica en el Perú, donde el modelo de desarrollo económico ha generado consecuencias negativas como: subempleo, desempleo, bajo ingreso per cápita que genera pobreza extrema, las cuales son el origen de múltiples factores que condicionan la permanencia del foco infeccioso de la enfermedad como: el déficit de saneamiento ambiental, hacinamiento, poca accesibilidad a los servicios de salud, deficiente educación para la salud, mala alimentación y estrés social (1).

Actualmente vivimos en una sociedad de diversidad cultural, cada uno con diferentes costumbres y normas, en donde factores como el sexo, la edad, el grado de instrucción y el empleo cumplen un papel muy importante porque depende de ellos que exista una adecuada facilidad para obtener información sobre la enfermedad, además que cuenten con los medios necesarios para lograr la recuperación de su salud, estos factores permiten que los pacientes tomen una decisión adecuada sobre sus prácticas de autocuidado (6).

Durante el presente año en el Centro de Salud del Comité Local de Administración en Salud San Martín de Porres se viene atendiendo a 38 pacientes, de los cuales dos pa- 
cientes presentan tuberculosis extrapulmonar y 36 pacientes con tuberculosis pulmonar; de este último, 14 son varones, 16 mujeres, y 6 menores de edad pertenecientes al Esquema I. Durante el internado comunitario que realizamos en el Centro de Salud CLAS San Martín de Porres, se logró interactuar con los pacientes y se conversó con varios de ellos que manifestaron lo siguiente: «No quería que nadie se me acerque»; «No quería saber nada», «Solo tomo un té y pan en el desayuno»; «Solo tomo desayuno y almuerzo»; «A veces no tengo tiempo para el almuerzo porque tengo mucho trabajo y solo ceno». También se observó que los pacientes no siempre se cubrían la boca al momento de toser, no conocían el nombre de las pastillas pero sí la cantidad y el color de las pastillas que recibían, desconocían en qué fase de la enfermedad se encontraban y de cuántas fases cuenta el esquema; en su ficha médica se observó que en algunos pacientes no había ganancia de peso, y cuando asistían a la toma de medicamentos supervisado no se observaba una adecuada higiene corporal y de vestimenta.

En las visitas domiciliarias se observó que la mayoría de pacientes no cuentan con una adecuada ventilación e iluminación en sus viviendas; en otros casos, los pacientes compartían su habitación con más de dos personas; incluso, toda la familia compartía una sola habitación. Además expectoraban y escupían directamente al suelo. Algunos pacientes abandonaron su tratamiento, y en otros casos se ausentaban por algunos días, al momento de preguntarles el porqué ellos manifestaban que era «Por su trabajo», pues salían de sus casas muy temprano y regresaban tarde. El personal encargado de la estrategia en el centro de salud es el licenciado en Enfermería pero, por falta de personal, algunas funciones las asume el personal técnico de Enfermería, el cual interactúa de forma constante con el paciente, y es quien resuelve sus dudas sobre la enfermedad de manera general.

Por lo mencionado, la tuberculosis pulmonar es calificada como un problema de trascendencia social, fundamento que exige la participación efectiva de cada uno de los pacientes, sus familias y comunidad para su erradicación. Es así que, por todo ello, En esta investigación se plantea identificar el nivel de conocimiento sobre las prácticas de autocuidado y su asociación con las características sociodemográficas en pacientes con diagnóstico de tuberculosis pulmonar - CLAS - Los Olivos 2012.

\section{MATERIAL Y MÉTODOS}

Estudio de tipo descriptivo de corte transversal, se realizó en el Centro de Salud CLAS «San Martín de Porres» ubicado en el distrito de Los Olivos, provincia y departamento de Lima - Perú. La institución está catalogada como un establecimiento de salud de nivel de atención I, que brinda servicios como promoción y prevención en las estrategias sanitarias de salud (inmunizaciones, prevención y control de enfermedades metaxénicas y otras transmitidas por vectores, prevención y control de tuberculosis, salud sexual y salud reproductiva, prevención y control de daños no transmisibles, alimentación y nutrición saludable), además de consultas externas en medicina general y psicología.

Se trabajó con toda la población que estuvo constituida por 30 pacientes con diagnóstico de tuberculosis pulmonar pertenecientes al esquema de tratamiento 1 que se encontraban registrados en la Estrategia Nacional de Prevención y Control de Tuberculosis Pulmonar del Centro de Salud Comité Local de Administración de Salud San Martín de Porres 2012.

Se consideró como criterios de exclusión a pacientes que ingresaron a la Estrategia Sanitaria de Tuberculosis Pulmonar una vez comenzada la recolección de datos, paciente multidrogorresistente y pacientes que abandonaron el tratamiento.

Para la recolección de datos se utilizó como técnica la encuesta, y como instrumento el cuestionario estructurado. El cuestionario fue elaborado por las investigadoras, se inició por la identificación de las dimensiones, luego los indicadores, y después los ítems; el instrumento constó de tres partes: la primera es la introducción, donde se menciona la presentación, el objetivo, la importancia de su participación y el agradecimiento. La segunda corresponde a los datos generales. La tercera consta de las preguntas referidas a los indicadores. El cuestionario, está estructurado con preguntas cerradas y de elección múltiple y fue calificado de la siguiente manera: 1 respuesta correcta; 0 respuesta incorrecta.

La validación del instrumento se llevó a cabo mediante el juicio de diez expertos; participaron profesionales con amplia trayectoria y conocedores del tema, cuyas opiniones y recomendaciones sirvieron para mejorar el instrumento. Los resultados de la evaluación fueron analizados mediante la prueba binomial, y se obtuvo valores de significancia estadística inferior a 0,05 para cada uno de los criterios de evaluación, lo que evidencia la validez del instrumento $(\mathrm{p}<0,05)$. Para obtener la confiabilidad del instrumento se realizó la prueba piloto a quince pacientes que fueron escogidos de forma aleatoria y que pertenecen a la Estrate- 
gia Nacional de Prevención y Control de Tuberculosis Pulmonar del centro de salud CLAS Laura Caller, Para el caso de la confiabilidad, el estadístico Alfa de Crombach resultó igual a 0,617 , lo que demuestra que el instrumento es confiable (Alfa $>0,6)$.

Para llevar a cabo la recolección de datos se coordinó con el enfermero encargado de la Estrategia Nacional de Prevención y Control de Tuberculosis Pulmonar para asistir al Centro de Salud en los días de toma de medicamentos durante un mes. Después de la toma de medicamento supervisado, en el mismo consultorio, se informó a cada paciente sobre el estudio; seguidamente, se hizo entrega de la hoja de consentimiento informado para la aceptación y su respectiva firma; luego se realizó la aplicación del instrumento a los pacientes de forma individualizada. Así mismo, es relevante mencionar que las investigadoras durante todo este proceso tomaron en cuenta las medidas de bioseguridad y confidencialidad de la información. Para finalizar, se brindó a la población educación sanitaria individualizada en el tema «Practicas de autocuidado en tuberculosis pulmonar». Al concluir la educación se entregó un tríptico con la información expuesta.

Los datos fueron codificados e ingresados a una base de datos creado en el programa estadístico SPSS versión 15.0 (Programa estadístico para las Ciencias Sociales) para su análisis.

Para determinar el nivel de conocimiento de las prácticas de autocuidado en pacientes con tuberculosis pulmonar registrados en la estrategia nacional de prevención y control de tuberculosis pulmonar se obtuvo tablas de distribución de frecuencia para las dimensiones de tratamiento farmacológico, alimentación y medidas de bioseguridad en el hogar según sus indicadores; las prácticas de autocuidado se clasificaron en niveles de bajo, medio y alto, según la escala de Estatinos.

Se determinó la relación entre el nivel de conocimiento sobre prácticas de autocuidado y las características sociodemográficas. Se obtuvieron tablas de contingencia donde las filas estuvieron constituidas por las características sociodemográficas ,y las columnas por los niveles de la variable conocimiento. La prueba que se utilizó para explorar dicha asociación fue la prueba chi cuadrado, la cual determinó la asociación entre las variables conocimiento de las prácticas de autocuidado y datos generales cunado se obtuvo un valor de significancia estadística inferior a $(\mathrm{p}<0,05)$.

El proyecto de investigación fue aprobado por el Comité
Institucional de Ética de la Universidad Peruana Cayetano Heredia. Se solicitó consentimiento informado a todos los participantes del estudio y la información fue manejada exclusivamente por las investigadoras conservando el anonimato de los participantes.

\section{RESULTADOS}

En la Tabla 1 se observa que el 43,3\% de los pacientes tiene un nivel de conocimientos medio. El $30 \%$ de los pacientes con TBC, tuvo un nivel de conocimiento alto. La tabla 2, muestra que el nivel de conocimientos que pre-

Tabla 1. Nivel de conocimientos de la práctica de autocuidado en el Centro de Salud Clas San Martín de Porres - 2012

\begin{tabular}{lcc}
\hline \multicolumn{1}{c}{ Nivel } & Número & \% \\
\hline Alto & 9 & 30,0 \\
Medio & 13 & 43,3 \\
Bajo & 8 & 26,7 \\
Total & 30 & 100,0 \\
\hline
\end{tabular}

Tabla 2. Nivel de conocimiento de las prácticas de autocuidado sobre el tratamiento farmacológico, alimentación y bioseguridad en el Centro de Salud Clas San Martín de Porres - 2012

\begin{tabular}{|c|c|c|c|}
\hline & Nivel & Número & $\%$ \\
\hline \multirow{3}{*}{ 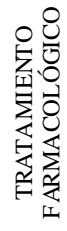 } & Alto & 7 & 23,3 \\
\hline & Medio & 18 & 60,0 \\
\hline & Bajo & 5 & 16,7 \\
\hline \multirow{3}{*}{ 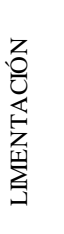 } & Alto & 7 & 23,3 \\
\hline & Medio & 18 & 60,0 \\
\hline & Bajo & 5 & 16,7 \\
\hline \multirow{4}{*}{ 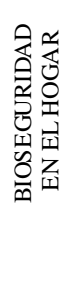 } & Alto & 6 & 20,0 \\
\hline & Medio & 19 & 63,3 \\
\hline & Bajo & 5 & 16,7 \\
\hline & Total & 30 & 100,0 \\
\hline
\end{tabular}


Tabla 3. Características sociodemográficas en el Centro de Salud CLAS San Martín de Porres - 2012.

\begin{tabular}{lrr}
\hline \multicolumn{1}{c}{$\begin{array}{c}\text { Datos } \\
\text { Socio demográficos }\end{array}$} & Número & $\%$ \\
\hline Edad & & \\
20 a 24 años & 5 & 16,7 \\
25 a 44 años & 15 & 50,0 \\
45 a 64 años & 7 & 23,3 \\
65 a mas & 3 & 10,0 \\
Sexo & & \\
Masculino & 14 & 46,7 \\
Femenino & 16 & 53,3 \\
Grado de instrucción & & \\
Primaria & 4 & 13,3 \\
Secundaria & 18 & 60,0 \\
Sup. técnica & 6 & 20,0 \\
Sup. universitaria & 2 & 6,7 \\
Trabaja & & \\
Sí & 20 & 66,7 \\
No & 10 & 33,3 \\
\hline
\end{tabular}

dominó en la dimensión tratamiento farmacológico, alimentación y bioseguridad con el 50,0\% ( $\mathrm{n}=15)$, seguido del nivel de conocimiento medio con el 33,3\% ( $\mathrm{n}=10)$, mientras que el nivel de conocimientos bajo solo alcanzó el $16,7 \%(\mathrm{n}=6)$.

El nivel de conocimientos que predominó en la dimensión alimentación fue el medio con el 60,0\% ( $\mathrm{n}=18)$, seguido del nivel de conocimiento alto con el 23,3\% $(n=7)$. El nivel de conocimientos bajo solo alcanzó el 16,7\% ( $\mathrm{n}=5)$.

El nivel de conocimientos que predominó en la dimensión bioseguridad en el hogar fue el medio con el 63,3\% ( $n=19)$, seguido del alto con el 20,0\% ( $\mathrm{n}=6)$. El nivel de conocimientos bajo solo alcanzó el 16,7\% ( $\mathrm{n}=5)$.

En la tabla 3, se aprecia que el $50 \%$ de los pacientes tiene entre 25 a 44 años, mientras que en menor proporción fue el de 65 a más años con el 10,0\% (n=3).

Respecto al sexo el femenino presentó el 53,3\% ( $\mathrm{n}=16)$, mientras el sexo masculino un $46,7 \%$ ( $n=14)$.

Con relación al grado de instrucción predominó el grado

Tabla 4. Asociación entre el nivel de conocimientos de prácticas de autocuidado con las características sociodemográficas: edad, sexo, grado de instrucción y ocupación en el Centro de Salud CLAS San Martín de Porres - 2012

\begin{tabular}{lccccccc}
\hline \multicolumn{1}{c}{ Sexo } & \multicolumn{3}{c}{ Niv el de conocimientos } & \multicolumn{2}{c}{ Total } \\
\hline Masculino & $\mathbf{N}^{\mathbf{0}}$ & Bajo & $\mathbf{N}^{\mathbf{0}}$ & Medio & $\mathbf{N}^{\mathbf{0}}$ & Alto & $\begin{array}{c}\text { \% } \\
\text { Femenino }\end{array}$ \\
Total & 2 & $42,9 \%$ & 6 & $42,9 \%$ & 2 & $14,3 \%$ & 100,0 \\
& 8 & $12,5 \%$ & 7 & $43,8 \%$ & 7 & $43,8 \%$ & 100,0 \\
\hline
\end{tabular}

Exacta de Fisher $=4,529, \mathrm{p}=0,132$

\begin{tabular}{llrrrrrr}
\hline \multicolumn{1}{c}{ Edad } & $\mathbf{N}^{\mathbf{1}}$ & \multicolumn{1}{c}{ Bajo } & $\mathbf{N}^{\mathbf{0}}$ & Medio & $\mathbf{N}^{\mathbf{0}}$ & Alto & $\begin{array}{c}\text { Total } \\
\mathbf{\%}\end{array}$ \\
\hline 20 a 24 años & 0 & 0 & 4 & $80,0 \%$ & 1 & $20,0 \%$ & 100,0 \\
25 a 44 años & 4 & $26,7 \%$ & 6 & $40,0 \%$ & 5 & $33,3 \%$ & 100,0 \\
45 a 64 años & 2 & $28,5 \%$ & 2 & $28,6 \%$ & 3 & $42,9 \%$ & 100,0 \\
65 a más & 2 & $66,7 \%$ & 1 & $33,3 \%$ & 0 & $0 \%$ & 100,0 \\
Total & 8 & $26,7 \%$ & 13 & $43,3 \%$ & 9 & $30,0 \%$ & 100,0 \\
\hline
\end{tabular}

Exacta de Fisher $=5,717, p=0,477$ 


\begin{tabular}{lccccccc}
\hline \multicolumn{1}{c}{ Instrucción } & $\mathbf{N}^{\mathbf{0}}$ & Bajo & $\mathbf{N}^{\mathbf{0}}$ & Medio & $\mathbf{N}^{\mathbf{0}}$ & Alto & $\begin{array}{c}\text { Total } \\
\%\end{array}$ \\
\hline Primaria & 4 & $100,0 \%$ & 0 & $0 \%$ & 0 & $0 \%$ & 100,0 \\
Secundaria & 3 & $16,7 \%$ & 8 & $44,4 \%$ & 7 & $38,9 \%$ & 100,0 \\
Superior técnica & 1 & $16,7 \%$ & 4 & $66,7 \%$ & 1 & $16,7 \%$ & 100,0 \\
Superior univ, & 0 & $0 \%$ & 1 & $50,0 \%$ & 1 & $50,0 \%$ & 100,0 \\
Total & 8 & $26,7 \%$ & 13 & $43,3 \%$ & 9 & $30,0 \%$ & 100,0 \\
\hline
\end{tabular}

Exacta de Fisher $=10,601, \mathrm{p}=0,035$

\begin{tabular}{lccccccc}
\hline \multicolumn{1}{c}{ Ocupación } & $\mathbf{N}^{\mathbf{0}}$ & Bajo & $\mathbf{N}^{\mathbf{0}}$ & Medio & $\mathbf{N}^{\mathbf{0}}$ & Alto & $\begin{array}{c}\text { Total } \\
\mathbf{\%}\end{array}$ \\
\hline Sí & 5 & $25,0 \%$ & 6 & $30,0 \%$ & 9 & $45,0 \%$ & 100,0 \\
No & 3 & $30,0 \%$ & 7 & $70,0 \%$ & 0 & $0 \%$ & 100,0 \\
Total & 8 & $26,7 \%$ & 13 & $43,3 \%$ & 9 & $30,0 \%$ & 100,0 \\
\hline
\end{tabular}

Exacta de Fisher $=7,382, \mathrm{p}=0,028$

de instrucción secundaria con un 60\% ( $\mathrm{n}=18)$, seguido de superior técnica con el $20,0 \%(n=6)$.

El 66,7\% (n=20) de los pacientes trabajan mientras que un $33,3 \%(n=10)$ no realiza ningún trabajo.

En la tabla 4, el 43,8\% (n=7) de los pacientes de sexo femenino presentó alto nivel de conocimientos, mientras que para el sexo masculino dicho porcentaje fue del 14,3\% $(n=2)$.

El $80 \%(n=4)$ y $40 \%(n=6)$ de los pacientes entre 20 a 24 y 25 a 44 años, presentaron un nivel de conocimiento medio; en el 42,9 $(n=3)$ de los pacientes entere 45 y 64 años predominó al nivel de conocimiento alto. El 66,7\% $(\mathrm{n}=2)$ de los pacientes de más de 65 años tuvieron un nivel de conocimiento bajo.

El 50,0\% (n=1) de pacientes con grado de instrucción superior universitaria presentó nivel de conocimientos alto, mientras que para el nivel de instrucción primaria el $100 \%$ $(n=4)$ presentaron pacientes nivel de conocimientos bajo.

El 45,0\% (n=9) de los pacientes que trabajan presentó un nivel de conocimiento alto, mientras que el $30 \%(n=3)$ de los pacientes que no trabajan presentó un nivel de conocimiento bajo.

\section{DISCUSIÓN}

La tuberculosis es una enfermedad con una condición altamente trascendente en salud pública; no solo por su elevada frecuencia en países en vías de desarrollo, sino también por las repercusiones económicas y sociales que esta tiene, debido a su efecto sobre la población económicamente activa (1).

En la tabla 1 se observa la información sobre el nivel de conocimientos relacionada con las prácticas de autocuidado en pacientes con tuberculosis que, en su mayoría, se encontró entre un nivel medio a bajo (70\%).

En el caso de la tuberculosis pulmonar, el autocuidado es importante, puesto que se requiere de prácticas adecuadas para evitar complicaciones y consecuencias de la enfermedad. Son importates aquellas prácticas de autocuidado relacionadas con los estilos de vida como alimentación, tratamiento farmacológicos y cuidados en el hogar. El autocuidado pone énfasis primordialmente en el rol del usuario con tuberculosis pulmonar, a través de sus prácticas de autocuidado, donde se convierte en el eje central para lograr recuperar y mantener su salud, además de prevenir la enfermedad con su entorno (5).

La estrategia utilizada por el Ministerio de Salud para pre- 
venir la enfermedad es a través de la información, educación y comunicación (7) que permite al paciente adquirir conocimientos sobre este tema; labor que debería ser reforzada por el enfermero encargado de la Estrategia Sanitaria Control de la Tuberculosis en el CLAS «San Martín de Porres» información que debe compartir con objetivo de disminuir la incidencia y prevalencia de esta enfermedad en la comunidad.

La carencia de conocimientos genera comportamientos errados y valorizaciones equivocadas. Con respecto al proceso salud-enfermedad la mayoría de los pacientes que tiene tuberculosis carecen de medios económicos, y del respaldo educativo básico para poder tomar decisiones oportunas y adecuadas. Por lo tanto, se puede deducir que la mayoría de los pacientes, al presentar un nivel de conocimientos de medio o bajo están en riesgo al presentar limitaciones en la aplicación de medidas preventivas en relación a la enfermedad, predisponiendo de esta manera al agravamiento de la enfermedad, al incremento del riesgo de contagio a los demás miembros de la familia, comunidad y el mayor riesgo de mortalidad.

En la tabla 2, se muestra que el nivel de conocimientos que predominó en la dimensión tratamiento fue el alto con el 50,0\% ( $\mathrm{n}=15)$, y el otro 50\% fue la suma del nivel medio y bajo; teniendo el nivel medio un $33,3 \%(n=10)$, mientras que el nivel de conocimientos bajo sólo alcanzó el $16,7 \%(n=6)$, este resultado difiere del estudio de Tacuri, quien encontró que el nivel de conocimientos sobre el tratamiento de la tuberculosis en la mayoría es de nivel me$\operatorname{dio}(8)$.

Lam, en su estudio de nivel de conocimiento sobre tuberculosis pulmonar en pacientes que reciben tratamiento para Tuberculosis en el Centro de Salud San Cosme - La Victoria del 2010 menciona que el $89,7 \%$ presenta un nivel de conocimiento sobre tratamiento para tuberculosis entre bajo y medio (9). Así mismo, en el estudio realizado por Antón y Mezones se encontró que la gran mayoría obtuvo un conocimiento medio y bajo (10).

El conocimiento viene a ser un factor determinante en la conducta de cada paciente frente a la aceptación del tratamiento y el mejor seguimiento de las indicaciones terapéuticas. En la investigación realizada en el CLAS «San Martín de Porres», la mitad de la población presentó un nivel de conocimientos alto, lo que podría hacer deducir que estos pacientes tienen una mayor aceptación hacia el tratamiento y, por lo tanto, tendrían un mejor seguimiento en las indicaciones terapéuticas, mientras que los pacientes que se encuentran entre el nivel de conocimientos de medio a bajo tendrán limitaciones en el consumo regular de los medicamentos.

Cabe resaltar que los pacientes también abandonan el tratamiento por falta de información de las reacciones adversas de los medicamentos que consume, por ejemplo, les puede dar ardor en el estómago, picazón en la piel, ictericia; el profesional de salud al no ser específico en cuanto a la información del tratamiento farmacológico, el paciente tendrá dudas, por lo tanto, podría ser un punto de quiebre para no seguir con el tratamiento.

El nivel de conocimiento de las prácticas de autocuidado sobre alimentación fue medio con un $60,0 \%(n=18)$, seguido del nivel de conocimiento alto con un $23,3 \%(n=7)$. El nivel de conocimientos bajo sólo alcanzó el 16,7\% (n=5). Este resultado coincide con Huamaní y et al., en su estudio «Estilos de vida de los adolescentes con tuberculosis pulmonar en el 2009», donde se encontró que el 52,6\% consume una dieta balanceada (11).

La alimentación en los pacientes se ve alterada, ya que la enfermedad es de curso prolongado, la tos y las respiraciones profundas incrementan la demanda de energía, disminuyendo su curva ponderal y en su mayoría los pacientes están desnutridos al inicio de la enfermedad; es preciso que en el hogar los pacientes cubran las necesidades básicas de ingestión de los nutrientes necesarios para cubrir sus demandas (12).

El Ministerio de Salud a través del Programa de Alimentación y Nutrición para el paciente ambulatorio con tuberculosis y familia (PANTBC), forma parte del conjunto de intervenciones del Estado peruano para apoyar la alimentación y recuperación del paciente ambulatorio con tuberculosis y proteger a su familia (13).

El Programa de Alimentación y Nutrición para el paciente ambulatorio con tuberculosis pulmonar, además de las acciones educativas y de evaluación nutricional, otorga a los beneficiarios una canasta de alimentos que cubre aproximadamente el $28 \%$ de los requerimientos calóricos y el $38 \%$ de los requerimientos proteicos de una familia compuesta por un paciente y dos contactos (14). En el Centro de Salud CLAS San Martín se les brinda una asesoría nutricional a cargo de los profesionales de salud, además, se les hace entrega de una canasta de víveres que contienen los siguientes alimentos: leche, aceite, cereales menestra, y conserva de pescado. Cabe resaltar que solo tiene derecho de recibir su canasta de víveres los pacientes que cumplen con el tratamiento farmacológico que se brinda gratuitamente en el centro de salud, a 
pesar de contar con estos beneficios algunos pacientes no recogen sus víveres y no asisten a la consejería nutricional. El nivel de conocimiento de autocuidado en alimentación fue medida en $60 \%$ de los pacientes.

El nivel de conocimiento de las prácticas de autocuidado sobre bioseguridad en el hogar predominó el nivel medio con $63,3 \%(n=19)$, seguido del alto con el $20,0 \%(n=6)$, el nivel de conocimientos bajo solo alcanzó el 16,7\% $(n=5)$. Estos resultados coinciden con el estudio realizado por Antón y Mezones, quienes encontraron que el 89\% estaba en este nivel pero con tendencia bajar (9); de igual manera, Lam encontró que el $62,9 \%$ presentó un nivel de conocimiento sobre cuidados en el hogar en tuberculosis bajo (8).

La tuberculosis pulmonar es una enfermedad infecciosa, que requiere diversos cuidados para que sea efectiva la recuperación del paciente (15). Por eso, es necesario que los pacientes posean conocimientos acerca de los cuidados en el hogar, cuidado al toser para prevenir contagio, medidas de cuidado en la expectoración, ventilación en el hogar, importancia de la habitación personal, medidas de higiene y prevención del hacinamiento $(16,17)$.

La mayoría de los pacientes con tuberculosis carecen de medios económicos y el respaldo educativo necesario para mantener un cuidado óptimo en el hogar. Por lo tanto, se puede deducir que el alto porcentaje de pacientes que poseen un nivel de conocimiento entre medio y bajo probablemente no realizarán los cuidados necesarios en el hogar, lo cual incide negativamente en la recuperación de los pacientes.

La tabla 3 muestra las características sociodemográficas, con referencia a la edad; el grupo etario que predominó fue el de 25 a 44 años. Este resultado coincide con el estudio realizado por Antón y Mezones, al encontrar que la edad promedio fue de 38,7 $\pm 4,16$ años (9).

Respecto al sexo el femenino presentó el 53,3\%. Estos resultados difieren del estudio de Antón y Mezones, al encontrar que el $65 \%$ fue de sexo masculino (9). Con relación al grado de instrucción predominó el grado de instrucción secundaria con un $60 \%$. Este resultado coincide con el estudio realizado por Antón y Mezones, encontrando un número de años académicos aprobados fue de 9,69 \pm 1,2 (9); sobre la ocupación el 66,7\% se encontró trabajando. Este resultado coincide con el estudio realizado por Antón y Mezones, al encontrar que el $61 \%$ estuvo laborando (9).
Los hallazgos obtenidos ponen en evidencia datos epidemiológicos similares a los estudios en el país sobre la tuberculosis donde da cuenta que en grupo afectado se encuentra entre la población adulto joven de regular nivel educativo, representado por la clase trabajadora. Actualmente, se vive en una sociedad de diversidad cultural, cada uno con diferentes costumbres y normas, y en donde los actores como el sexo, la edad, el grado de instrucción y el empleo cumplen un papel importante porque depende de ellos que exista una adecuada facilidad para obtener información sobre la enfermedad; además, que cuenten con los medios necesarios para lograr la recuperación de su salud, estos factores permiten que los pacientes tomen una decisión adecuada sobre sus prácticas de autocuidado.

En la tabla 4 se observa que el conocimiento de prácticas de autoconocimiento no presenta asociación con el sexo de los pacientes. Este resultado es similar a lo hallado por Antón y Mezones en donde no se encontró asociación entre ambas variables (9). Cabe resaltar que en la investigación a pesar que no se encontró asociación significativa, el porcentaje del sexo femenino predominó, lo cual solo refleja la realidad del Centro de Salud CLAS San Martín, el resultado puede variar en las próximas investigaciones que se realicen (9).

En la información brindada por el Ministerio de Salud la distribución de los casos con tuberculosis pulmonar según edad, fluctuan entre los 20 a 59 años (18). Teniendo en cuenta que en la investigación realizada en el centro de salud el 50\% de los pacientes tienen entre 25 a 44 años, podrimos decir que se trata de una población joven que se inicia de la PEA. Adicionalmente a ello este grupo es el que tiene mejor nivel de conocimiento en comparación a los otros grupos etarios, lo que reflejaría que este grupo adulto joven están concientizados por el mismo problema de salud que afrontan y, por lo mismo, buscan información para su pronta recuperación.

El conocimiento de prácticas asociado con el grado de instrucción presenta asociación significativa, cabe resaltar que solo hay dos pacientes de grado de instrucción superior.

Por lo tanto, se puede deducir que las personas que tienen grado de instrucción superior buscan más información sobre su enfermedad y tienen mayor interés, mientras que en los pacientes con grado de instrucción primaria presentan un nivel de conocimientos bajo, estos pacientes podrán no aplicar medidas preventivas en relación a la enfermedad, dado su bajo nivel de conocimiento que, predisponiendo de esta manera al agravamiento de la enfer- 
medad e incremento de la predisposición de contagio a los demás miembros de la familia y comunidad, y el mayor riesgo de mortalidad.

El conocimiento de prácticas asociado con la ocupación presenta asociación significativa. Este resultado difiere de Antón y Mezones que no se encontró asociación en ambas variables.

El conocimiento en el trabajo es un conjunto colectivo de saber y saber hacer $(19,20)$ es la fuente de la competitividad de los trabajadores, es lo que les proporciona su capacidad de adaptación y competitividad en el mercado. Los trabajadores con conocimientos tienen mayor accesibilidad a la información, debido al enriquecimiento producido por los intercambios, desarrollo social y creatividad. En cuanto a la investigación, los pacientes que trabajan presentaron un nivel de conocimiento alto ya que tienen mayor desarrollo de vida social, y son los que podrían buscar más información para recuperar su estado de salud y prevenir el contagio hacia sus familiares y entorno de trabajo.

\section{REFERENCIAS BIBLIOGRÁFICAS}

1. Organización Mundial de la Salud. Media Centre Tuberculosis Fact Sheet N. ${ }^{\circ} 104$ [Internet]. Ginebra: OMS; 2010. [citado 05 Ene 2011]. Disponible en: http:/ /www.who.int/mediacentre/factsheets/fs 104/es/ index.html.

2. Organización Mundial de la Salud. Tuberculosis (TB) Un mundo sin tuberculosis [Internet]. Ginebra: OMS; 2010. [citado 15 Nov 2010]. Disponible en: http:// www.who.int/tb/es/index.html

3. Organización Mundial de la Salud. Estadísticas Sanitarias Mundiales [Internet]. Ginebra: OMS; 2010 [citado 07 Feb 2011]. Disponible en URL: http:// www.who.int/whosis/whostat/ES_WHS10_Full.pdf.

4. Subirana K. No hay distrito de Lima que se encuentre libre de la Tuberculosis [Internet]. El Comercio; 23 de Agosto de 2011. [citado 01 Feb 2012]. Disponible en: http://elcomercio.pe/lima/1149808/noticia-no-haydistrito-lima-que-se-encuentre-libre-tuberculosis.

5. Vásquez P. Autocuidado para el manejo de Tuberculosis Pulmonar en el Programa de Departamento de Medicina Preventiva [tesis]. Lima; Universidad Nacional San Marcos, 2008.

6. Tobon O. El autocuidado: Una habilidad para vivir. RPS [Internet]. 2010 Febrero [citado 28 Feb 2012]. Disponible en: http://promocionsalud.ucal das.edu.co/ downloads/Revista\%208_5.pdf.
7. MINSA. Estrategia Sanitaria Nacional de Tuberculosis de la Dirección General de Salud de las Personas [Internet]. Ministerio de Salud; Perú; 2010. [citado 1 Feb 2012]. Disponible en: http://www.minsa. gob.pe/portada/esntbc_default.asp.

8. Tacuri ME. Relación entre adherencia y nivel de conocimientos sobre el tratamiento de los pacientes con tuberculosis en El Centro de Salud Primavera El Agustino [tesis]. Lima: Universidad Nacional Mayor de San Marcos; 2009.

9. Lam AL. Nivel de conocimientos sobre tuberculosis pulmonar en pacientes que recibieron tratamiento para tuberculosis en el Centro de Salud San Cosme La Victoria [tesis]. Lima: Universidad Nacional Mayor de San Marcos; 2010.

10. Antón C, Mezones A. Nivel de conocimientos y actitud hacia el tratamiento de la Tuberculosis en el Centro de Salud Sicchez [tesis]. Piura: Universidad Nacional de Piura; 2009.

11. Huamani C, Jáuregui C, Mendoza V. Estilos de vida de los adolescentes con tuberculosis pulmonar que asisten a la estrategia sanitaria de TBC al Establecimiento de la Red de Puente Piedra [tesis]. Lima: Universidad Peruana Cayetano Heredia; 2009.

12. Rodríguez R. Fisiología de la Respiración. $5^{\circ}$ ed. Volumen 2. Ed. Harcourt S. A. Madrid. España. 2004.

13. Dieta sana para pacientes con TBC. Diario El Peruano. 2008 Marzo 25; Actualidad: pág. 20.

14. Nutrinet Perú. Programas Sociales. Perú; 2011. [Internet]. [citado 24 Ene 2011]. Disponible en: http:/ /peru.nutrinet.org/politicas-publicas/programassociales.

15. Comisión honoraria para la lucha antituberculosa y enfermedades prevalentes. Programa nacional de control de tuberculosis. [Internet]. [citado 14 Dic 2011]. Disponible en: http://www.chlaep.org.uy/ programas-control-tuberculosis-preguntasfrecuentes.php.

16. Técnicas básicas de enfermería; 2009 [Internet]. [citado 1 Dic 2012] Disponible en: http://books.google. com.pe/books?id=8sMRpy-thPEC\&pg=PA47\&lpg $=$ PA $47 \& \mathrm{dq}=$ TECNICAS+BASICAS+DE+ENFERMERIA +CADA+CUANTO+SE+CAMBIA+LA+PIJAMA\&source =bl\&ots=R6vbs1z0NT\&sig=H613s0Of-SJfdmvjEag AKB92AU\&hl=es\&sa $=$ X\&ei $=4 \mathrm{hGmUqmgCI} 2 \mathrm{ekQeG}$

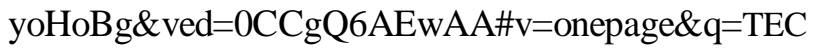
NICAS\%20BASICAS\%20DE\%20ENFERMERIA\%20CADA $\% 20$ CUANTO $\% 20$ SE\%20CAMBIA\%20LA\%20PIJAMA\&f $=$ false

17. Hábitos de higiene; 2009. [Internet]. [citado 11 May 2009]. Disponible en: http://loshabitosdehigiene. blogspot.com/. 
18. Jave O. National Strategy of Tb Perú. PERU; 2011. [Internet]. [citado 10 Abr 2013]. Disponible en: http:/ /www.imtunmsm.epiredperu.net/eventos/aniver sario2011/cursotb/ponencias/01_Jave_TB_Peru.pdf.

19. Henric M. La gestión del conocimiento y los equipos de trabajo. México; 2013 [Internet]. [citado 12 Abr 2013]. Disponible en: http://www.sht.com.ar/archivo/ Management/gestion_conoc.htm

20. Instituto de salud del estado de México [Internet].México: [actualizado 24 Feb 2012; citado 18 Jun 2011]. Disponible en: http://salud.edo mex.gob.mx/html/article.php?sid=168.

\section{Correspondencia}

Doris Velásquez Carranza

Miguel Baquero 251. Lima

Correo electrónico: doris.velasquez@ upch.pe

Télefono: 4314731 -4244731

Forma de citar este artículo: Aliaga-Rojas G, Alvarado-Herrera M, Alva-Vera Á, Velásquez-Carranza D. Conocimientos sobre prácticas de autocuidado y su asociación con las características sociodemográficas en pacientes con diagnóstico de tuberculosis pulmonar, CLAS San Martín de Porres - Los Olivos 2012. Rev enferm Herediana. 2013;6(1):02-11. 